\title{
A Geometric Snake Model for Segmentation of Medical Imagery
}

\author{
Anthony Yezzi, Jr., Member, IEEE, Satyanad Kichenassamy, Arun Kumar, \\ Peter Olver, and Allen Tannenbaum,* Member, IEEE
}

\begin{abstract}
In this note, we employ the new geometric active contour models formulated in [25] and [26] for edge detection and segmentation of magnetic resonance imaging (MRI), computed tomography (CT), and ultrasound medical imagery. Our method is based on defining feature-based metrics on a given image which in turn leads to a novel snake paradigm in which the feature of interest may be considered to lie at the bottom of a potential well. Thus, the snake is attracted very quickly and efficiently to the desired feature.
\end{abstract}

Index Terms - Active contours, active vision, edge detection, gradient flows, segmentation, snakes.

\section{INTRODUCTION}

$\mathbf{T}$ HE technique of snakes or active contours has become quite popular for a variety of applications in the past few years. This methodology is based upon the utilization of deformable contours which conform to various object shapes and motions. Snakes have been used for edge and curve detection, segmentation, shape modeling, and visual tracking. Active contours have also been widely applied for various applications in medical imaging. For example, snakes have been employed for the segmentation of myocardial heart boundaries as a prerequisite from which such vital information such as ejection-fraction ratio, heart output, and ventricular volume ratio can be computed. (See [20], [42], and the references therein.) In this paper, we will apply a new snake paradigm which the authors have developed [25], [26] for edge detection and segmentation of various kinds of medical imagery including magnetic resonance imaging (MRI), computed tomography (CT), and ultrasound.

In the classical theory of snakes, one considers energy minimization methods where controlled continuity splines are allowed to move under the influence of external image

Manuscript received August 31, 1995; revised October 14, 1996. This work was supported in part by the National Science Foundation under Grant DMS-9204192 and Grant ECS-9122106, in part by the Air Force Office of Scientific Research under Grant F49620-94-1-0058DEF, and in part by the Army Research Office under Grant DAAH04-94-G-0054 and Grant DAAH0493-G-0332. The Associate Editor responsible for coordinating the review of this paper and recommending its publication was J. S. Duncan. Asterisk indicates corresponding author.

A. Yezzi, Jr. is with the Department of Electrical Engineering, University of Minnesota, Minneapolis, MN 55455 USA.

S. Kichenassamy and P. Olver are with the Department of Mathematics, University of Minnesota, Minneapolis, MN 55455 USA.

A. Kumar is with the Department of Aerospace Engineering, University of Minnesota, Minneapolis, MN 55455 USA.

*A. Tannenbaum is with the Department Electrical Engineering, University of Minnesota, Minneapolis, MN 55455 USA (e-mail: tannenba@ee.umn.edu).

Publisher Item Identifier S 0278-0062(97)02399-9. dependent forces, internal forces, and certain constraints set by the user [7], [24], [55]. As is well known, there may be a number of problems associated with this approach such as initializations, existence of multiple minima, and the selection of the elasticity parameters. Moreover, natural criteria for the splitting and merging of contours (or for the treatment of multiple contours) are not readily available in this framework. (See, however, the recent solution to this problem proposed by McInerney and Terzopoulos [34].)

In this work, we will apply a new active contour method which was developed [25], [26]. (Independently, similar methods have been recently formulated [9], [49].) Our method unifies the curve evolution approaches for active contours [8], [32] and the classical energy methods mentioned above [7], [24], [55]. Since the geometric curve evolution equations can in fact treat merging and splitting of contours, our model gives the user the capability of automatically handling topological changes within the gradient flow energy framework. Moreover, our model has an important advantage over the geometric snakes of [8], [32] as well. Indeed, the approach in these works amounts to curve evolution together with a multiplicative stopping term. These models will only slow down the active contour at an edge, and so the snake will in general pass through the desired feature (see Sections II and IV for more discussion about these points). Our model handles the topological complexities while providing extra stopping power to the capture the features of interest, based on first principles from geometric energy minimization. (The geometric snakes of [8] and [32] are not derived from a minimization of energy.) Thus, the approach to active contours which we give below may be regarded as the natural unification of the two main snake models previously considered.

More precisely, our technique employs ideas from Euclidean curve shortening evolution which defines the gradient direction in which the Euclidean perimeter is shrinking as fast as possible; see Section III. We therefore note that we can derive new active contour models by multiplying the Euclidean arclength by a function tailored to the features of interest to which we want to flow and then writing down the resulting gradient evolution equations. Mathematically, this amounts to defining a new metric in the plane tailored to the given image and then computing the corresponding gradient flow. This leads to some new snake models which efficiently attract the given active contour to the features of interest (which basically lie at the bottom of a potential well). Further, the method allows us to naturally write down three-dimensional (3-D) active surface 
models for 3-D image segmentation which we do in [25], [26], and [59]. One can completely justify this method using viscosity theory which is done as well in [25] and [26] (some remarks regarding some of the salient points in this regard are made in the Appendix).

The contents of this paper may be summarized as follows. In Section II, we briefly sketch some background from the classical theory of snakes. In Section III, we give the relevant from curve evolution theory, which allows us to formulate our new active contour paradigm in Section IV. In Sections $\mathrm{V}$ and VI, we indicate how these methods may be extended for volumetric segmentation based on mean curvature flow. Finally in Section VII, we apply our methods to some specific medical images, and then we draw our conclusions in Section VIII. We also include an Appendix with some of the relevant mathematical details for the convenience of the reader.

\section{BACKGROUND ON SNAKES}

In this section, we briefly review the energy based optimization approach to deformable contours as discussed in [7], [12], [24], and [55]. For complete details, we refer the interested reader to the collection of papers in [7, especially [54]].

Let $C(p)=(x(p), y(p))^{T}$ be a closed contour in $\mathbf{R}^{2}$ where $0 \leq p \leq 1$. (Note that the superscript $T$ denotes transpose.) We now define an energy functional on the set of such contours ("snakes"), $\mathcal{E}(C)$. Following standard practice, we take $\mathcal{E}(C)$ to be of the form

$$
\mathcal{E}(C)=\mathcal{E}_{\text {int }}(C)+\mathcal{P}(C)
$$

where $\mathcal{E}_{\text {int }}$ is the internal deformation energy and $\mathcal{P}$ is an external potential energy which depends on the image. (Other external constraint forces may be added.) Perhaps the most common choice for the internal energy is the quadratic functional

$$
\mathcal{E}_{\mathrm{int}}(C):=\int_{0}^{1} w_{1}(p)\left\|C_{p}\right\|^{2}+w_{2}(p)\left\|C_{p p}\right\|^{2} d p
$$

where $w_{1}$ and $w_{2}$ control the "tension" and "rigidity" of the snake, respectively. (Note that the subscripts denote derivatives with respect to $p$ in the latter expression, and $\|\cdot\|$ denotes the standard Euclidean norm.)

Let $I:[0, a] \times[0, b] \rightarrow \mathbf{R}^{+}$be the given grey-scale image. Then the external potential energy depends on the image $I(x, y)$. It can be defined by

$$
\mathcal{P}(C):=\int_{0}^{1} P(C(p)) d p
$$

where $P(x, y)$ is a scalar potential function defined on the image plane. The local minima of $P$ attract the snake. For example, we may choose $P$ to be

$$
P(x, y):=c\left\|\nabla G_{\sigma} * I(x, y)\right\|
$$

for a suitably chosen constant $c$, in which case the snake will be attracted to intensity edges. Here $G_{\sigma}$ denotes a Gaussian smoothing filter of standard deviation $\sigma$.

Solving the problem of snakes amounts to finding, for a given set of weights $w_{1}, w_{2}$, the curve $C$ that minimizes
$\mathcal{E}$. As argued in [8], the classical snakes method provides an accurate location of the edges sufficiently near a given initialization of the curve, it has the ability to extract smooth shapes, and can retrieve angles. (Of course, one must tune the parameters $w_{1}, w_{2}$ to the given problem.) On the other hand, it does not directly allows simultaneous treatment of multiple contours. The classical (energy) approach of snakes cannot deal with changes in topology, unless special topology handling procedures are added [34], [52]. The topology of the initial curve will be the same as the one of the (possibly wrong) final solution. This is the basic formulations of twodimensional (2-D) active contours. Other related and 3-D formulations have been proposed in the literature (e.g., [12], [13], and [56]). Reviewing all of them is beyond the scope of the present paper. We should add that there have been a number of nice papers which improve the traditional snake methodology. (See [58], [21], and the references therein).

One also typically considers dynamic time-varying models in which $C(p)$ becomes a function of time as well; see [54]. In this case, one defines a kinetic energy and the corresponding Lagrangian (the difference between the kinetic energy and the energy $\mathcal{E}$ defined above). Applying the principle of least action, one derives the corresponding Lagrange equation which one tries to solve numerically employing various approximations.

In the approach to be given below in Section IV, we will also use an energy method. However, in contrast to more ad hoc approaches, we believe that our energy is intrinsic to the given geometry of the problem, as is the corresponding gradient flow. It will also be able to handle topological changes in a completely automatic fashion.

\section{Curve Evolution Theory}

The mathematical foundation of our new active contour model is based on Euclidean curve shortening. Since this is essential in understanding our proposed snake models, we will now sketch the some of the key concepts from curve evolution theory in the plane $\mathbf{R}^{2}$.

For $\kappa$ the curvature, and $\overrightarrow{\mathcal{N}}$ the inward unit normal, one considers families of plane curves flowing according to the geometric heat equation

$$
\frac{\partial C}{\partial t}=\kappa \overrightarrow{\mathcal{N}}
$$

This equation has a number of properties which make it very useful in image processing. In fact, it has become the basis of a nonlinear scale-space for shape representation [2], [4], [27], [28].

In particular, (1) is the Euclidean curve shortening flow, in the sense that the Euclidean perimeter shrinks as quickly as possible when the curve evolves according to (1) [17], [19]. Since, we will need a similar argument for the snake model we discuss in Section IV, let us work out the details.

Let $C=C(p, t)$ be a smooth family of closed curves where $t$ parametrizes the family and $p$ the given curve, say $0 \leq p \leq 1$. (Note we assume that $C(0, t)=C(1, t)$ and similarly for the first derivatives). Define the length functional

$$
L(t):=\int_{0}^{1}\left\|\frac{\partial C}{\partial p}\right\| d p .
$$


Then differentiating (taking the "first variation"), and using integration by parts, we see that

$$
\begin{aligned}
L^{\prime}(t) & =\int_{0}^{1} \frac{\left\langle\frac{\partial C}{\partial p}, \frac{\partial^{2} C}{\partial p \partial t}\right\rangle}{\left\|\frac{\partial C}{\partial p}\right\|} d p \\
& =-\int_{0}^{1}\left\langle\frac{\partial C}{\partial t}, \frac{1}{\left\|\frac{\partial C}{\partial p}\right\|} \frac{\partial}{\partial p}\left[\frac{\frac{\partial C}{\partial p}}{\left\|\frac{\partial C}{\partial p}\right\|}\right]\left\|\frac{\partial C}{\partial p}\right\|\right\rangle d p .
\end{aligned}
$$

But observing now that

$$
\left\|\frac{\partial C}{\partial p}\right\| d p=: d s
$$

is the arc-length, and using the definition of curvature, the last integral is

$$
-\int_{0}^{L(t)}\left\langle\frac{\partial C}{\partial t}, \kappa \overrightarrow{\mathcal{N}}\right\rangle d s
$$

Hence, we see

$$
L^{\prime}(t)=-\int_{0}^{L(t)}\left\langle\frac{\partial C}{\partial t}, \kappa \overrightarrow{\mathcal{N}}\right\rangle d s
$$

Thus, the direction in which $L(t)$ is decreasing most rapidly is when

$$
\frac{\partial C}{\partial t}=\kappa \overrightarrow{\mathcal{N}}
$$

Thus, (1) defines a gradient flow.

A much deeper fact is that simple closed curves converge to "round" points when evolving according to (1) without developing singularities. This means that if we consider an associated family of dilated curves of constant area (look at the evolving family of shrinking curves under a "magnifying glass"), the curves of the family approach a circle; see [17] and [19]. This fact is the basis for the nonlinear geometric scale-spaces studied in vision recently [2], [4], [27], [28], as well as the active contour models considered below.

In Section IV, we will see that a level set representation of (2) will be utilized in all the active contour models. Roughly, this is an evolution of the graph of a function all of whose level sets evolve according to (2). How to associate such a level set representation will be sketched in the Appendix. See [38], [46] and [47] for all the details. For future reference, the level set version of (1) is given by the function $\Psi(x, y, t)$ evolving according to

$$
\frac{\partial \Psi}{\partial t}=\|\nabla \Psi\| \operatorname{div}\left(\frac{\nabla \Psi}{\|\nabla \Psi\|}\right) .
$$

(See Section B of the Appendix.)

\section{New Active Contour Paradigm}

In two key papers, Caselles et al. [8] and Malladi et al. [32] formulate snake models based on the level set interpretation of the Euclidean curve shortening equation. Indeed, their model is

$$
\frac{\partial \Psi}{\partial t}=\phi(x, y)\|\nabla \Psi\|\left(\operatorname{div}\left(\frac{\nabla \Psi}{\|\nabla \Psi\|}\right)+\nu\right) .
$$

Here, the function $\phi(x, y)$ depends on the given image and is used as a "stopping term." For example, the function $\phi(x, y)$ may be defined in such a way that it has very small magnitude in the vicinity of an edge and so acts to stop the evolution when the contour gets close to an edge. In [8] and [32], the term

$$
\phi:=\frac{1}{1+\left\|\nabla G_{\sigma} * I\right\|^{n}}
$$

is chosen, where $I$ is the (grey-scale) image and $G_{\sigma}$ is a Gaussian (smoothing) filter. (In [8], $n=1$, and in [32], $n=2$ ). The function $\Psi(x, y, t)$ evolves in (4) according to the associated level set flow for planar curve evolution in the normal direction with speed a function of curvature which was introduced in the fundamental work of Osher-Sethian [37], [38], [45], [46], [47].

As we have just seen, the Euclidean curve shortening part of this evolution, namely

$$
\frac{\partial \Psi}{\partial t}=\|\nabla \Psi\| \operatorname{div}\left(\frac{\nabla \Psi}{\|\nabla \Psi\|}\right)
$$

may be derived as a gradient flow for shrinking the perimeter as quickly as possible using only local information. As is explained in [8], the constant inflation term $\nu$ is added in (4) in order to keep the evolution moving in the proper direction. Note that we are taking $\Psi$ to be negative in the interior and positive in the exterior of the zero level set contour.

The inflationary constant may be considered both with a positive sign (inward evolution of the evolution of the contour in the direction of decreasing $\Psi$ ) and with a negative sign (outward or expanding evolution). (Note, the sign convention we have taken for $\Psi$ above.) In the latter case, this can be referred to as expanding "balloons" or "bubbles" [12], [53]. One should also note that there are many possibilities for a stopping term besides intensity: texture, optical flow, stereo disparity, etc.

The models proposed in [8] and [32] have the important advantage over the classical models discussed in Section II of being able to automatically handle different topologies. On the other hand, (4) does not come from the minimization of an energy functional as in the classical model. In fact, for inward evolutions, (4) consists of terms which shrink the initial contour (the inflation and the curvature term), and the stopping term consisting of $\phi$. Thus, the model seems a bit $a d$ hoc and the powerful energy intuition of the classical case is lacking. From a more practical point of view, unless the stopping term is exactly zero (which never occurs in real images), the evolving contour will not in general completely stop at the given edge. (There is an extensive mathematical discussion of this in [26]; see also, the Appendix.) We would therefore like a model with additional stopping power as well.

Therefore, we would like to find a model which combines the advantages of both the classical energy and the geometric curve evolution models. This is precisely the type of model we are about to give. The idea is to find an energy functional based on first principles and right down the corresponding flow. This will lead to a new curve evolution method which will unify these two fundamental methods.

Our approach is based on modifying the model equation (4) using the gradient evolution ideas given in Section III. We accomplish this, by changing the ordinary Euclidean arc-length 
function along a curve $C=(x(p), y(p))^{T}$ with parameter $p$ given by

$$
d s=\left\|C_{p}\right\| d p=\left(x_{p}^{2}+y_{p}^{2}\right)^{1 / 2} d p
$$

to

$$
d s_{\phi}=\phi d s=\left(x_{p}^{2}+y_{p}^{2}\right)^{1 / 2} \phi d p
$$

where $\phi(x, y)$ is a positive differentiable function. The resulting metric is called a conformal metric and appears in a variety of applications including phase transitions. (See [25] and the references therein.)

We now modify the computation made in Section III, i.e., we compute the gradient flow for curve shortening relative to the new metric $d s_{\phi}$. Consequently, we set

$$
L_{\phi}(t):=\int_{0}^{1}\left\|\frac{\partial C}{\partial p}\right\| \phi d p .
$$

Then, taking the first variation of the modified length function $L_{\phi}$, and using integration by parts just as above, we get that

$$
-L_{\phi}^{\prime}(t)=\int_{0}^{L_{\phi}(t)}\left\langle\frac{\partial C}{\partial t}, \phi \kappa \overrightarrow{\mathcal{N}}-(\nabla \phi \cdot \overrightarrow{\mathcal{N}}) \overrightarrow{\mathcal{N}}\right\rangle d s
$$

which means that the direction in which the $L_{\phi}$ perimeter is shrinking as fast as possible is given by

$$
\frac{\partial C}{\partial t}=(\phi \kappa-\nabla \phi \cdot \overrightarrow{\mathcal{N}}) \overrightarrow{\mathcal{N}}
$$

[See the Appendix for all of the mathematical details involved in the derivation of (6)]. This is precisely the gradient flow corresponding to the minimization of the length functional $L_{\phi}$. The level set implementation of this flow may be computed to be

$$
\frac{\partial \Psi}{\partial t}=\phi\|\nabla \Psi\| \operatorname{div}\left(\frac{\nabla \Psi}{\|\nabla \Psi\|}\right)+\nabla \phi \cdot \nabla \Psi .
$$

This evolution attracts the contour very quickly to the feature which lies at the bottom of the potential well described by the gradient flow (7). As in [8] and [32], we may also add a constant inflation term, and so derive a modified model of (4) given by

$$
\frac{\partial \Psi}{\partial t}=\phi\|\nabla \Psi\|\left(\operatorname{div}\left(\frac{\nabla \Psi}{\|\nabla \Psi\|}\right)+\nu\right)+\nabla \phi \cdot \nabla \Psi .
$$

Notice that for $\phi$ as in (5), $\nabla \phi$ will look like a "doublet" near an edge. (An edge is defined by a step function, and $\nabla \phi$ involves two derivatives of the grey-scale image function $I$.) One may easily check that the effect of $\nabla \phi$ is to attract the evolving contour as it approaches an edge, and to push the contour back out if it should pass the edge [25], [26]. Thus, the model we have, combines that of [8] and [32] together with the extra stopping power derived from the new gradient term. We should also note that (8) was derived using first principles from energy minimization exactly as in the standard snakes approaches (see [7] and the references therein).

Of course, one may choose other candidates for $\phi$ in order to pick out other features as we remarked above. The point is that the metric $d s_{\phi}$ has the property that it becomes small where $\phi$ is small and vice versa. Thus, at such points lengths decrease and so one needs less "energy" in order to move. Consequently, it seems that such a metric is natural for attracting the deformable contour to an edge when $\phi$ has the form (5).

We have implemented this snake model based on the algorithms of Osher-Sethian [37], [38], [45], [46], [47] and Malladi et al. [32]. In Section VII, we apply (8) to a number of medical imaging modalities.

\section{Mean Curvature Surface Evolution}

It is easy to extend the 2-D active contour model just presented to the 3-D case. The key to our segmentation approach is a modification of the ordinary area functional, and the corresponding gradient flow. In order to motivate this, we need to briefly summarize some of the literature on mean curvature motion and the resulting theory of minimal surfaces. For all the key concepts in differential geometry, we refer the reader to [14].

Let $S:[0,1] \times[0,1] \rightarrow \mathbf{R}^{3}$ denote a compact embedded surface with (local) coordinates $(u, v)$. Let $H$ denote the mean curvature, that is, $H$ is the arithmetic mean of the principal curvatures. (Recall that at each point $p$, the surface $S$ has two principal curvatures given by the maximum and minimum of plane curves which are cut out on the surface by planes meeting it orthogonally at $p$.) We let $\overrightarrow{\mathcal{N}}$ denote the inward unit normal. Set

$$
S_{u}:=\frac{\partial S}{\partial u}, \quad S_{v}:=\frac{\partial S}{\partial v} .
$$

Then the infinitesimal area on $S$ is given by

$$
d S=\left(\left\|S_{u}\right\|^{2}\left\|S_{v}\right\|^{2}-\left\langle S_{u}, S_{v}\right\rangle^{2}\right)^{1 / 2} d u d v .
$$

It is a classical fact that the gradient flow associated to the area functional for surfaces can be defined in terms of the mean curvature (see [33] and the references therein). More precisely, for a family of surfaces depending on a parameter $t$, consider the area functional

$$
A(t):=\int_{0}^{1} \int_{0}^{1}\left(\left\|S_{u}\right\|^{2}\left\|S_{v}\right\|^{2}-\left\langle S_{u}, S_{v}\right\rangle^{2}\right)^{1 / 2} d u d v .
$$

Taking the first variation, and using integration by parts, it is easy to compute that

$$
\frac{d A}{d t}=-\iint_{S}\left\langle\frac{\partial S}{\partial t}, H \overrightarrow{\mathcal{N}}\right\rangle d S .
$$

Therefore, the direction in which the area is shrinking most rapidly (using only local information) is given by

$$
\frac{\partial S}{\partial t}=H \overrightarrow{\mathcal{N}}
$$

Consequently, this flow is very closely connected to the theory of minimal surfaces (surfaces of minimal area with given boundary conditions). 


\section{3-D ACtive Contour Models}

In this section, we will formulate our geometric 3-D contour models based on the mean surface motion sketched in Section V. Our method is derived by modifying the Euclidean area by a function which depends on the salient image features which we wish to capture.

Indeed, let $\phi: \Omega \rightarrow \mathbf{R}$ be a positive differentiable function defined on some open subset of $\mathbf{R}^{3}$. The function $\phi(x, y, z)$ will play the role of a "stopping" function. Thus, the function $\phi(x, y, z)$ will depend on the given grey-level image. Explicitly, the term $\phi(x, y, z)$ may chosen to be small near a 3-D edge, and so acts to stop the evolution when the 3$\mathrm{D}$ contour reaches the edge. For example, as in the 2-D case, we can choose

$$
\phi:=\frac{1}{1+\left\|\nabla G_{\sigma} * I\right\|^{2}}
$$

where $I=I(x, y, z)$ is the (grey-scale) volumetric image and $G_{\sigma}$ is a Gaussian (smoothing) filter.

What we propose to do is to replace the Euclidean area given above by a modified (conformal) area depending on $\phi$, namely

$$
d S_{\phi}:=\phi d S .
$$

Indeed, for a family of surfaces (with parameter $t$ ), consider the $\phi$-area functional

$$
A_{\phi}(t):=\iint_{S} d S_{\phi}
$$

Then, exactly as above, taking the first variation and using a simple integration by parts argument, we get that

$$
\frac{d A_{\phi}}{d t}=-\iint_{S}\left\langle\frac{\partial S}{\partial t},(\phi H-\nabla \phi \cdot \overrightarrow{\mathcal{N}}) \overrightarrow{\mathcal{N}}\right\rangle d S
$$

(See, the Appendix). The corresponding gradient flow is then

$$
\frac{\partial S}{\partial t}=(\phi H-\nabla \phi \cdot \overrightarrow{\mathcal{N}}) \overrightarrow{\mathcal{N}}
$$

Notice that Euclidean conformal area $d S_{\phi}$ is small near an edge. Thus, we would expect and initial 3-D contour to flow to the potential well indicated by the evolution (11).

The level set version of (11) [37], [38], and [47] is given in terms of the evolving level set function $\Psi(x, y, z, t)$ by

$$
\frac{\partial \Psi}{\partial t}=\phi\|\nabla \Psi\| \operatorname{div}\left(\frac{\nabla \Psi}{\|\nabla \Psi\|}\right)+\nabla \phi \cdot \nabla \Psi .
$$

As before, a constant inflation term $\nu$ may be added to give the model

$$
\Psi_{t}=\phi\|\nabla \Psi\|\left(\operatorname{div}\left(\frac{\nabla \Psi}{\|\nabla \Psi\|}\right)+\nu\right)+\nabla \phi \cdot \nabla \Psi .
$$

(Once again, this inflationary constant may be taken to be either positive (inward evolution) or negative in which case it would have an outward or expanding effect. As in the 2-D case, we take $\Psi$ to be negative in the interior and positive in the exterior of the zero level set.)

It is important to note that there is a mathematically a major difference between the 2-D and 3-D models discussed here.
Indeed, the geometric heat equation will shrink a simple closed curve to a round point without developing singularities, even if the initial curve is nonconvex. The geometric model equation (4) is based on this flow. For surfaces, it is well known that singularities may develop in the mean curvature flow (9) nonconvex smooth surfaces. (The classical example is the dumbbell.) We should note however that the mean curvature flow does indeed shrink smooth compact convex surfaces to round "spherical" points; see [22]. Because of these problems, several researchers have proposed replacing mean curvature flow by flows which depend on the Gaussian curvature $K$. Indeed, define $K_{+}:=\max \{K, 0\}$. Then Caselles and Sbert [10] have shown that the affine invariant flow

$$
\frac{\partial S}{\partial t}=\operatorname{sign}(H) K_{+}^{1 / 4} \overrightarrow{\mathcal{N}}
$$

will (smoothly) shrink rotationally symmetric compact surfaces to ellipsoidal shaped points. Thus, one could replace the mean curvature part by $\operatorname{sign}(H) K_{+}^{1 / 4}$ in (13). Another possibility would be to use $K_{+}^{1 / 2}$ as has been proposed in [35]. See also, [53]. (Note that Chow [11] has shown that convex surfaces flowing under $K^{1 / 2}$ shrink to spherical points.) These possible evolutions for 3-D segmentation have been discussed in our recent paper [59].

\section{Applications to Medical Imagery}

We will now apply the active contour model derived above to some medical imagery. The numerical methods we have used come from the level set evolution techniques developed by Osher-Sethian [37], [38], [45], [46], [47], and Malladi et al. [32]. To speed up the evolutions, we have used the local versions of these algorithms precisely as described in [1]. The equations described in this paper have been coded for the case of active contours on 2-D images. In Section A of the Appendix, we make some remarks about this method.

For 2-D active contours, the evolution equation as derived above is [(8)]

$$
\frac{\partial \Psi}{\partial t}=\phi\|\nabla \Psi\|\left(\operatorname{div}\left(\frac{\nabla \Psi}{\|\nabla \Psi\|}\right)+\nu\right)+\nabla \phi \cdot \nabla \Psi
$$

where $\nu$ is a constant inflation force and $\kappa:=\operatorname{div}\left(\frac{\nabla \Psi}{\|\nabla \Psi\|}\right)$ is the curvature of the level sets of $\Psi(x, y, t)$. This equation describes a propagating front, and we are interested in its propagation in the plane of an image. It is known that a propagating front may not remain smooth at all times (for example, it may cross itself). For evolution beyond the discontinuities the solutions are required to satisfy an entropy condition to ensure that the front remains physically meaningful at all times. The discrete approximations to the spatial derivatives are thus, derived from the entropy condition. Osher and Sethian [38] have given such entropy satisfying schemes and these have been used successfully in shape modeling [32]. We can regard a decomposition of our speed function as

$$
F(\kappa)=\nu+\operatorname{div}\left(\frac{\nabla \Psi}{\|\nabla \Psi\|}\right)=\nu+\kappa
$$



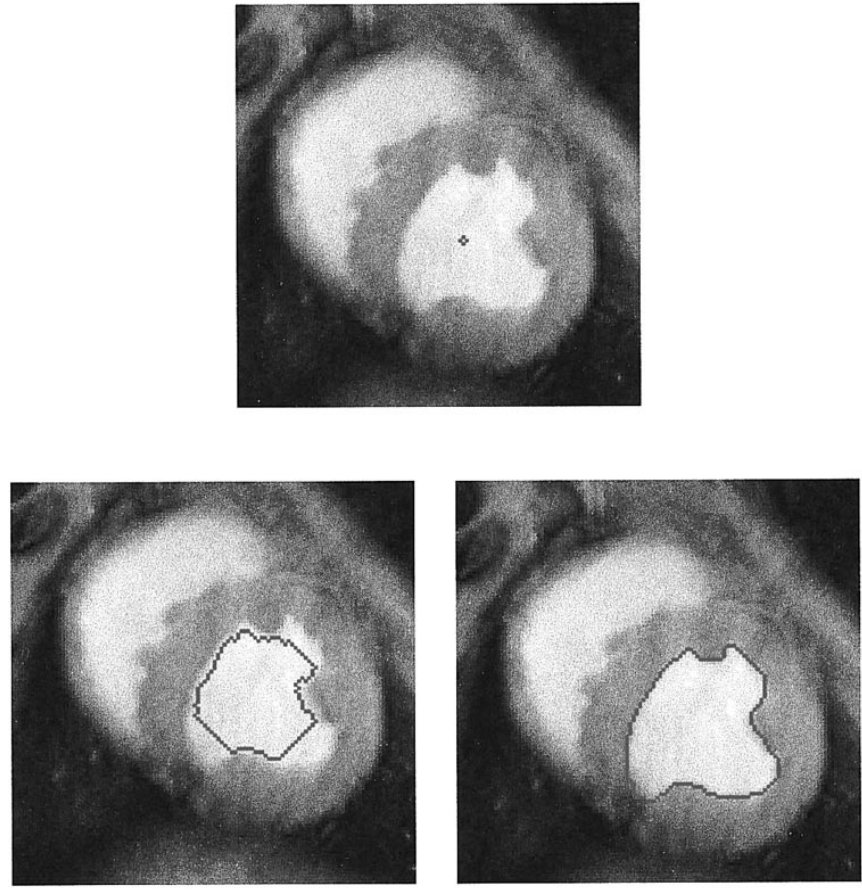

Fig. 1. Contour extraction from MRI heart image via bubble.
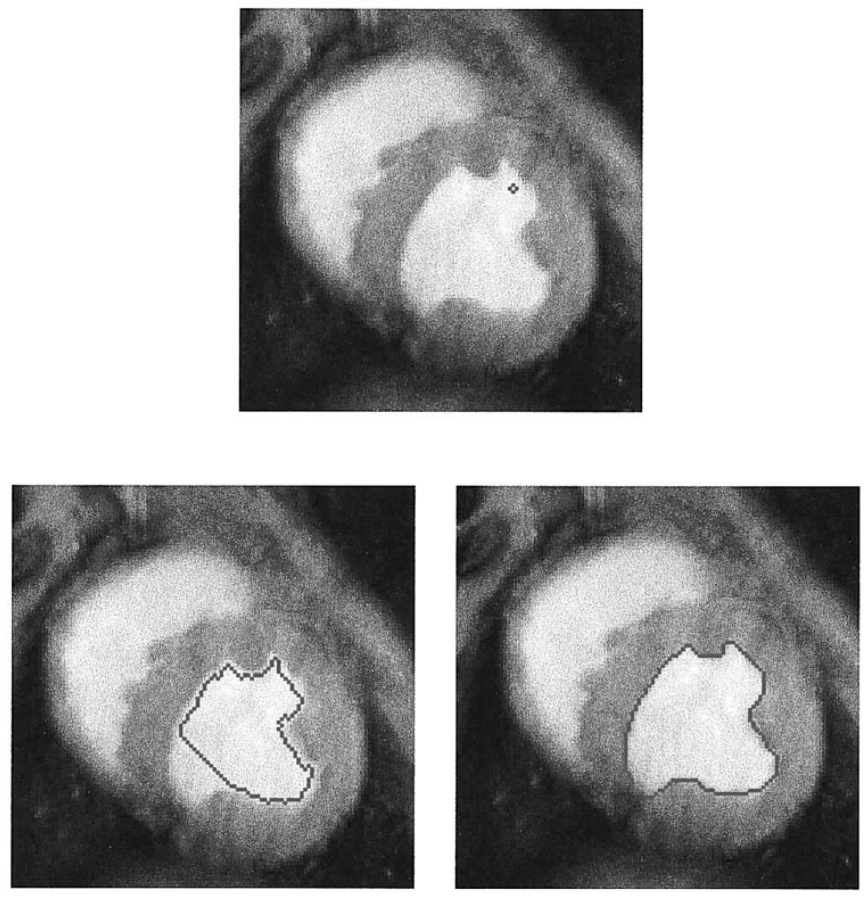

Fig. 2. Contour extraction from MRI heart image via different bubble placement.

where $\nu$ is regarded as the constant passive advection term and the curvature $\kappa$ is the diffusive term of the speed function. The inflation part in (8), i.e., $\nu \phi|| \nabla \Psi \|$ is approximated using upwind schemes. The diffusive part, i.e., $\kappa \phi|| \nabla \Psi \|$ is approximated using usual central differences [32].

There are several stability considerations for the choice of the step sizes. In [32], it is noted that for the evolution equation used in that work the requirement is $\Delta t=O\left(\Delta x^{2}\right)$.
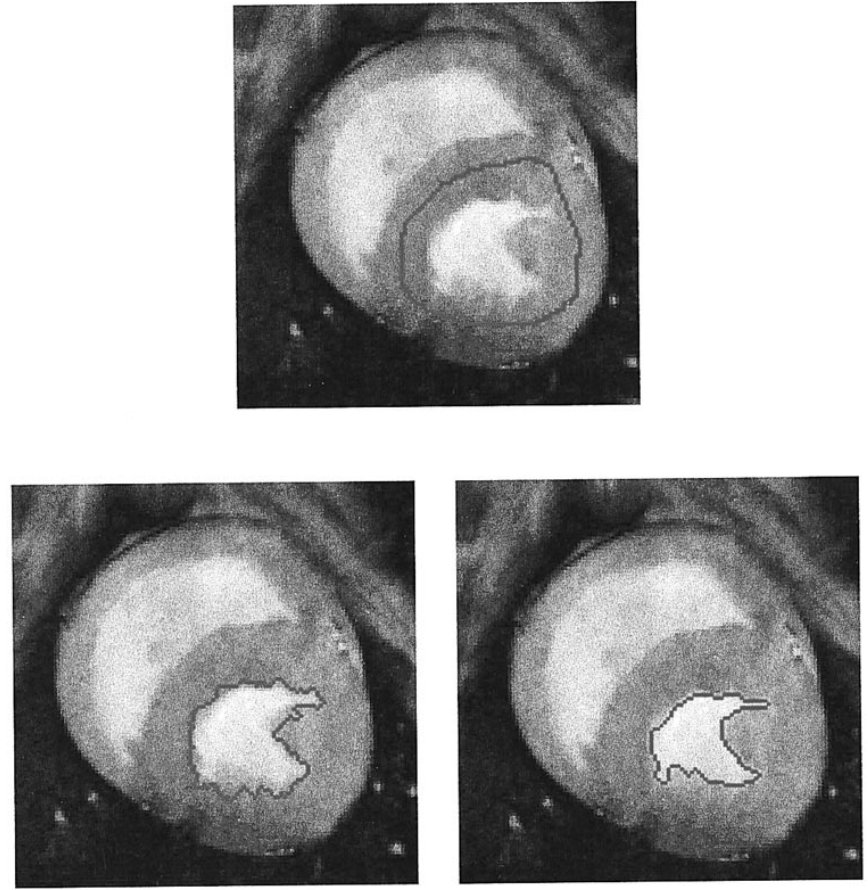

Fig. 3. Contour extraction from MRI heart image via snake.

Therefore, if small spatial step sizes are used, it forces a small time step and the resulting evolution can be very slow. One possibility for speeding up the evolution is to use a larger inflationary force and move the front faster (recall the advection term causes a constant contraction/expansion of the front). However, in our experience with using the approach in [32] this results in large motion of the front causing "overshooting" of the edge of the feature of interest in the image, because $\phi$ might not be rigorously zero on the desired contour. This problem is resolved by the evolution in (8) in which $\nabla \phi$ has a behavior similar to a doublet near an edge. Thus, it exerts a "stronger" stopping effect and arrests the evolution of the contour close to an edge. In our simulations, we have observed that this arresting behavior of the $\nabla \phi \cdot \nabla \Psi$ term allows use of large inflationary forces, resulting in features being extracted in relatively fewer time steps.

\section{A. Contour Extraction Results}

We now describe a number of 2-D images from which we extracted the contours using the snake/bubble technique which we have previously described. We have chosen images from three of the key modalities, MR, ultrasound, and CT to demonstrate our techniques. All of the simulations were done on a Sparc10 workstation. The differences in unit iteration times among the various images are functions of the initial contours as well as the type of image. For example, the number of iterations depends on how close the initial contour is placed to the region of interest (ROI). This is explicitly illustrated in Figs. 1 and 2. On the other hand, for numerous initial placements we were always successful in finding the relevant edges. 

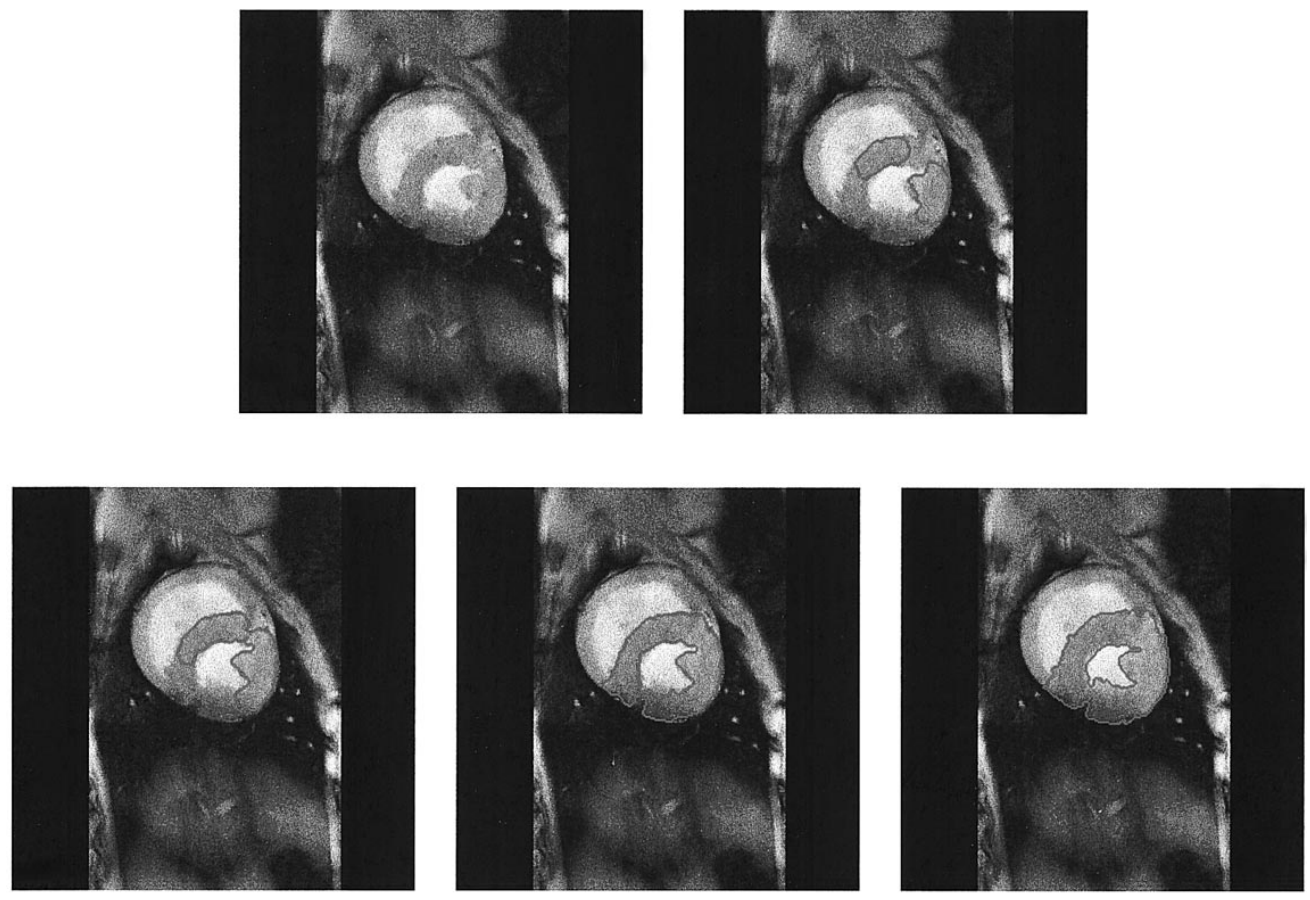

Fig. 4. Myocardial contour extraction via merging bubbles.
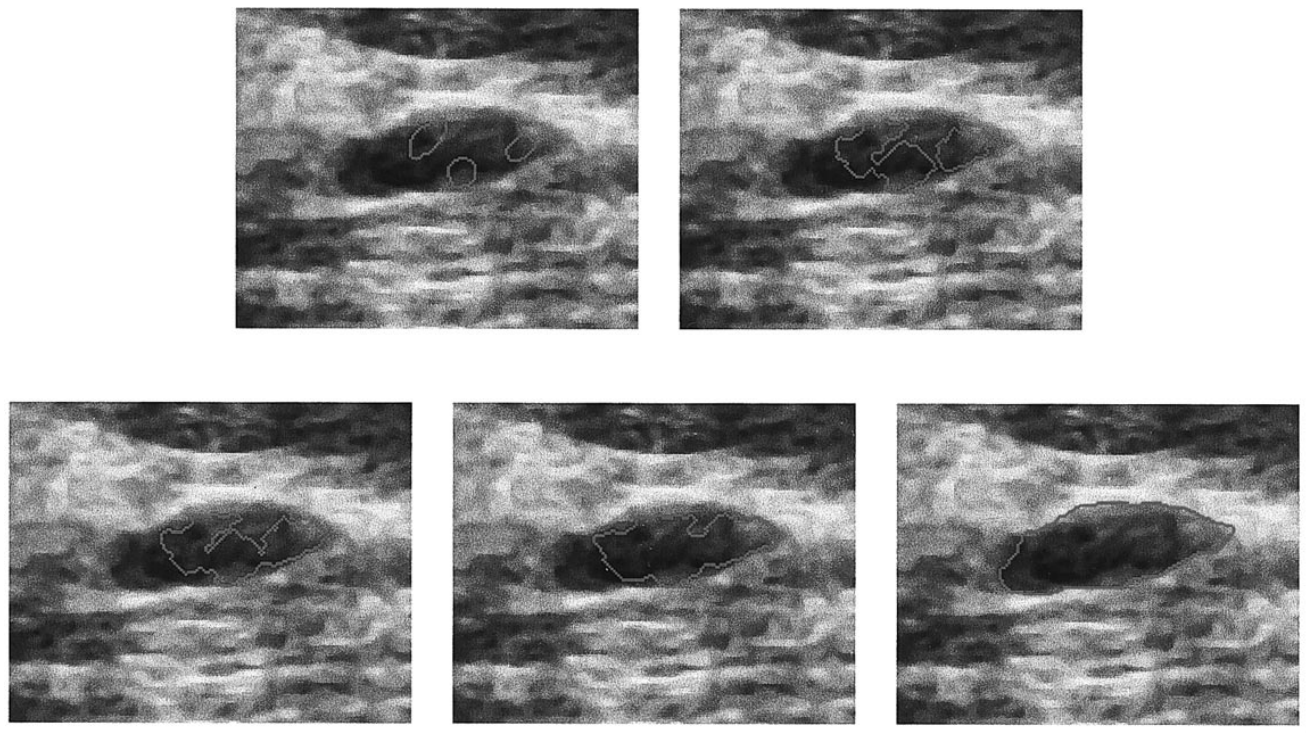

Fig. 5. Contour extraction of cyst from ultrasound breast image via merging bubbles.

The value we took for the inflation parameter $\nu$ in all of the extractions below was one.

1) In Fig. 1, using an initial bubble, we find the boundary of the left ventricle in an MRI heart image. The contour was found in 45 iterations which took about $3 \mathrm{~s}$.

2) In Fig. 2, we demonstrate the insensitivity of our methods to bubble placement. Therefore, using a very different placement of the bubble, we find once again the boundary of the left ventricle. The contour was found in about 60 iterations which ran for about $4 \mathrm{~s}$.

3) In Fig. 3, the snake (inward) evolution is utilized for the same purpose in another MRI heart image. The contour was found in 30 iterations which also ran for about $2.5 \mathrm{~s}$.

4) In Fig. 4, we place two bubbles to find the myocardium surrounding the left ventricle. Notice how the expanding bubbles automatically merge. The evolution took about 80 steps which ran for about $8.5 \mathrm{~s}$.

5) Fig. 5, illustrates bubbles capturing the edge of a cyst in a breast ultrasound image. We start with three bubbles in order to demonstrate once again the ability of the algorithm to automatically handle merging. Notice that we successfully find the contour in a very noisy environment. Because of the noise, we presmoothed the image using ten iterations of the affine curve shortening 

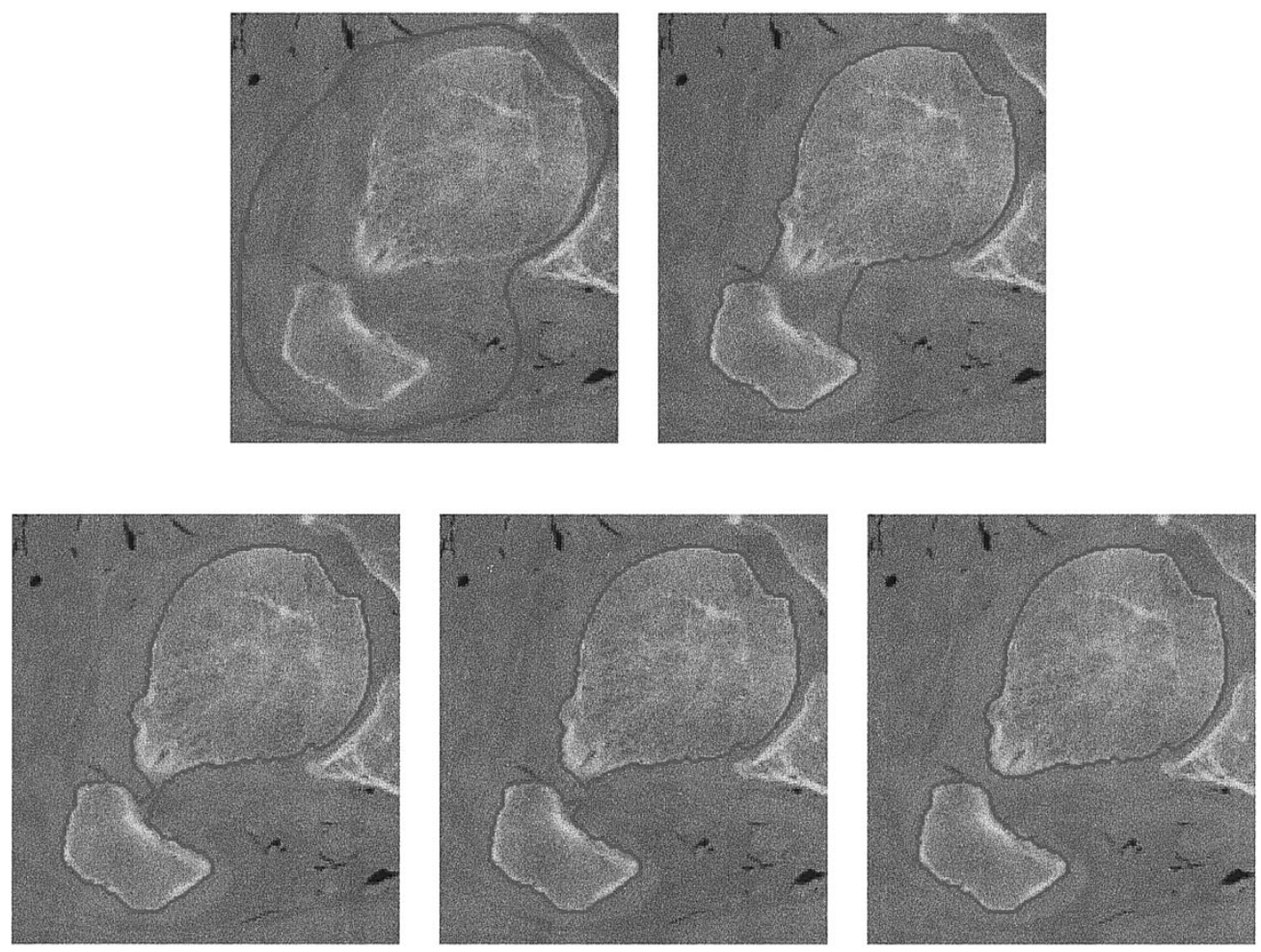

Fig. 6. Contour extraction from $\mathrm{CT}$ bone image via snake.

nonlinear filter [2], [3], [43], [44]. The cyst boundary was found in 75 steps which took about $5 \mathrm{~s}$.

6) In Fig. 6, we indicate the detection of a multiply connected contour in a CT bone image using inward evolving snakes. Notice how we get automatic splitting of the contour to catch the two bone regions. In this case, the evolution took about 67 steps which ran for $8 \mathrm{~s}$.

It is very interesting to compare the contour extraction results in Figs. 1-4 with similar MR cardiac images using standard snakes, e.g., in [20]. Using the traditional methodology one must start quite close to the contour of interest in order to be able to capture in. In Figs. 1-4 this is certainly not the case. Moreover, Figs. 1 and 2, illustrate the fact that initial bubbles placed quite far from one another still can extract the relevant contour.

The results from Figs. 4-6 demonstrate the speed and utility of our methods for treating topological changes, multiple contours, and finding boundaries for segmentation even in noisy environments. Because of the ease of simplicity of use and speed of the algorithm, it seems ideal for use on a wide variety of medical imagery as indicated above. For bubbles, just place the bubble in the ROI, and let it grow to capture the desired boundary. (For this technique to work in general, the initial bubble must be placed completely inside the given feature of interest.)

\section{CONCLUSION}

In this paper, we have applied the novel active contour model formulated in [25] and [26] to a number of medical images coming from a variety of common modalities. The power of this technique in extracting features from even rather noisy medical images has been demonstrated. Our approach is geometric, based on image-dependent Riemannian metrics and the associated gradient flows for active contour models. Fast, reliable implementations of the 3-D mean curvature equations for the volumetric segmentation of medical images are reported in [59].

\section{APPENDIX \\ MATHEMATICAL JUSTIFICATION OF THE MODELS}

In this Appendix, we will provide some mathematical details of the equations we study here. This section is optional and has been included in order to make this paper as self-contained as possible. Obviously, we can only sketch some of the relevant background here, and so we refer the interested reader to the papers referenced in our discussion below.

\section{A. Derivation of Active Contour Model}

First we will derive the model equation (6). We use the same notation as in Section IV. Accordingly, let $C=C(p, t)$ be a smooth family of closed curves where $t$ parametrizes the family and $p$ the given curve, say $0 \leq p \leq 1$. Taking the first variation of the length functional

$$
L_{\phi}(t):=\int_{0}^{1}\left\|C_{p}\right\| \phi d p
$$

we get that

$$
L_{\phi}^{\prime}(t)=\int_{0}^{1} \phi_{t}\left\|\frac{\partial C}{\partial p}\right\| d p+\int_{0}^{1} \phi\left\langle C_{p t}, C_{p}\right\rangle \frac{1}{\left\|C_{p}\right\|} d p .
$$


Set

$$
I_{1}=\int_{0}^{1}\left\|C_{p}\right\| \phi d p=\int_{0}^{L(t)}\left\langle\nabla \phi, C_{t}\right\rangle d s
$$

and

$$
\begin{aligned}
I_{2} & =\int_{0}^{1} \phi\left\langle C_{p t}, C_{p}\right\rangle \frac{1}{\left\|C_{p}\right\|} d p \\
& =-\int_{0}^{1}\left\langle C_{t},\left(\frac{\phi C_{p}}{\left\|C_{p}\right\|}\right)_{p}\right\rangle d p \quad \text { (integration by parts) } \\
& =-\int_{0}^{L(t)}\left\langle C_{t},(\phi \overrightarrow{\mathcal{T}})_{s}\right\rangle d s .
\end{aligned}
$$

Using the fact [33] that

$$
\overrightarrow{\mathcal{T}_{s}}=\kappa \overrightarrow{\mathcal{N}}
$$

we see

$$
I_{2}=-\int_{0}^{L(t)}\left\langle C_{t},(\nabla \cdot \overrightarrow{\mathcal{T}}) \overrightarrow{\mathcal{T}}+\phi \kappa \overrightarrow{\mathcal{N}}\right\rangle d s
$$

Therefore

$$
\begin{aligned}
L^{\prime}(t) & =I_{1}+I_{2} \\
& =-\int_{0}^{L(t)}\left\langle C_{t},(\phi \kappa-(\nabla \cdot \overrightarrow{\mathcal{N}})) \overrightarrow{\mathcal{N}}\right\rangle d s
\end{aligned}
$$

from which we derive, the required equation

$$
C_{t}=(\phi \kappa-(\nabla \cdot \overrightarrow{\mathcal{N}})) \overrightarrow{\mathcal{N}}
$$

The derivation of the mean curvature flow (11) in Section VI is very similar; see, [25] and [26].

\section{B. Level Set Representations}

We now go over the level set representation for curves flowing according to functions of the curvature. This is the basis of the numerical algorithm chosen for the active contour model given in Section VII. The level set approach for such evolutions is due to Osher and Sethian [38], [46], [47], [48]. Let $C(p, t): S^{1} \times[0, \tau) \rightarrow \mathbf{R}^{2}$ be a family of curves satisfying the following evolution equation:

$$
\frac{\partial C}{\partial t}=\beta(\kappa) \overrightarrow{\mathcal{N}} .
$$

There are a number of problems which must be solved when implementing curve evolution equations such as (16) on computer. For example, singularities may develop. A typical instance of this phenomenon is when $\beta \equiv 1$ in (16); here, even a smooth initial curve can develop singularities. The question is how to continue the evolution after the singularities appear. A natural way is to choose the solution which agrees with the Huygens principle [45], [46], or as Sethian observed, if the front is viewed as a burning flame, this solution is based on the principle that once a particle is burnt, it stays burnt [45], [46]. One can show that, from all the weak solutions corresponding to (16), the one derived from the Huygens principle is unique, and can be obtained via the entropy condition constraint.
In any numerical algorithm, we have the key requirements of accuracy and stability. The numerical algorithm must approximate the evolution equation, and it must be robust. Sethian [47] showed that a simple, Lagrangian, difference approximation, requires an impractically small time step in order to achieve stability. The basic problem with Lagrangian formulations is that the marker particles on the evolving curve come very close during the evolution.

The algorithm proposed by Osher and Sethian [38], [46], [47], [48] provides a reliable numerical solution for curve (and hypersurface) evolution. It is based on the Hamilton-Jacobi and viscosity theory. Indeed, first the curve is embedded in a 2-D surface, and then the equations of motion are solved using a combination of straightforward discretization, and numerical techniques derived from hyperbolic conservation laws.

The embedding step is done in the following manner: The curve $C(p, t)$ is represented by the zero level set of a smooth and Lipschitz continuous function $\Psi: \mathbf{R}^{2} \times[0, \tau) \rightarrow \mathbf{R}$. Assume that $\Psi$ is negative in the interior and positive in the exterior of the zero level set. We consider the zero level set, defined by

$$
\left\{X(t) \in \mathbf{R}^{2}: \Psi(X, t)=0\right\} .
$$

We have to find an evolution equation of $\Psi$, such that the evolving curve $C(t)$ is given by the evolving zero level $X(t)$, i.e.,

$$
C(t) \equiv X(t)
$$

By differentiating (17) with respect to $t$ we obtain

$$
\nabla \Psi(X, t) \cdot X_{t}+\Psi_{t}(X, t)=0
$$

Note that for the zero level, the following relation holds:

$$
\frac{\nabla \Psi}{\|\nabla \Psi\|}=-\overrightarrow{\mathcal{N}}
$$

In this equation, the left side uses terms of the surface $\Psi$, while the right side is related to the curve $\mathcal{C}$. The combination of (16) to (20) gives

$$
\Psi_{t}=\beta(\kappa)\|\nabla \Psi\|
$$

and the curve $C$, evolving according to (16), is obtained by the zero level set of the function $\Psi$, which evolves according to (21). Sethian [47] called this scheme an Eulerian formulation for front propagation, because it is written in terms of a fixed coordinate system. Finally, one can compute that

$$
\kappa=\operatorname{div}\left(\frac{\nabla \Phi}{\|\nabla \Phi\|}\right)
$$

(This is the curvature of an implicitly defined curve; see [33].) Therefore, we derive the level set representation of Euclidean curve shortening (3) as a special case of (21). 


\section{Viscosity Theory}

Next, we make some remarks about the existence and uniqueness of the models we consider in this paper. (Again full mathematical details may be found in [26].) The equations we study here are special cases of a nonlinear diffusion equation of the form

$$
\begin{array}{r}
\Psi_{t}=\Psi(x) \sum_{i, j=1}^{n} a^{i j}(\nabla \Psi) \frac{\partial^{2} \Psi}{\partial x_{i} \partial x_{j}}+H(x, \nabla \Psi), \\
x=\left(x_{1}, \ldots, x_{n}\right) .
\end{array}
$$

Because of the form of the $a^{i j}$ in the equations we consider, and the fact that $\Psi$ may vanish, studying (22) requires some care; in particular, the solutions are not expected to be sufficiently regular for the equation to make sense, and we need to use a type of generalized solutions known as viscosity solutions. Defining these is beyond the scope of this paper, and so we refer the interested reader to [23] for a general discussion of the theory and [26] for their application to the equations studied here. We will just summarize some of the relevant conclusions of [26] now.

The key point is that there exists a unique solution for (22) in a suitable viscosity sense. (See [26, Th. 1]). Moreover, slight differences between images will not become artificially enhanced by our active contour methods. We have also shown that the level curves of the function $\Psi$ do approach the desired contour $\Gamma=\{\Psi=0\}$. These results show that the model of the present paper is justified from a theoretical standpoint.

We should also note that if $\phi$ is not rigorously zero on the desired contour, the evolution has no reason to stop. In fact, one would rather expect it to shrink the snake to a point. This explains why in dealing with poor images, one may see the snake passing through the features of interest. This is a further advantage of the additional doublet stopping term $\nabla \phi$. Some care must therefore be given to the choice of $\phi$, so that the evolution slows down significantly near the desired contour.

Moreover, the appropriate amount of inflation of (8) can be estimated (from above). Without the gradient term, it is safe to take an inflation term of the order of the curvature of the desired contour. Finally, we can give precise estimates on sensitivity of the final contour as a function of initial contour placement (see [26, Theorem 2]).

\section{REFERENCES}

[1] D. Adalsteinsson and J. Sethian, "A fast level set method for propagating interfaces," J. Computat. Phys., vol. 118, pp. 269-277, 1995.

[2] L. Alvarez, F. Guichard, P. L. Lions, and J. M. Morel, "Axiomes et equations fondamentales du traitement d'images," C. R. Acad. Sci., vol. 315, pp. 135-138, 1992.

[3] - "Axioms and fundamental equations of image processing," Arch. Rational Mech., Anal., vol. 123, 1993.

[4] _ "Axiomatization et nouveaux operateurs de la morphologie mathematique," C. R. Acad. Sci., vol. 315, pp. 265-268, 1992.

[5] L. Alvarez, P. L. Lions, and J. M. Morel, "Image selective smoothing and edge detection by nonlinear diffusion," SIAM J. Numer. Anal., vol. 29, pp. 845-866, 1992.

[6] L. Alvarez and J. M. Morel, "Formalization and computational aspects of image analysis," Dept. Inform. Syst., Univ. de las Palmas de Gran Canaria, Rep. 0493, 1993.

[7] A. Blake and A. Yuille, Active Vision,. Cambridge, MA: MIT, 1992.

[8] V. Caselles, F. Catte, T. Coll, and F. Dibos, "A geometric model for active contours in image processing," Numerische Mathematik, vol. 66, pp. 1-31, 1993.
[9] V. Caselles, R. Kimmel, and G. Sapiro, "Geodesic snakes," in Proc. ICCV, June 1995.

[10] V. Caselles and C. Sbert, "What is the best causal scale-space for 3D images?," Dept. Math. Comput. Sci., Univ. Illes Balears, Palma de Mallorca, Spain, Tech. Rep., Mar. 1994.

[11] B. Chow, "Deforming convex hypersurfaces by the $n$th root of the Gaussian curvature," J. Differential Geometry, vol. 22, pp. 117-138, 1985.

[12] L. D. Cohen, "On active contour models and balloons," CVGIP: Image Understanding, vol. 53, pp. 211-218, 1991.

[13] I. Cohen and L. D. Cohen, "Using deformable surfaces to segment 3-D images and infer differential structure," CVGIP: Image Understanding, vol. 56, pp. 242-263, 1992.

[14] M. P. Do Carmo, Differential Geometry of Curves and Surfaces. Englewood Cliffs, NJ: Prentice-Hall, 1976.

[15] 1992.

[16] C. L. Epstein and M. Gage, "The curve shortening flow," in Wave Motion: Theory, Modeling, and Computation, A. Chorin and A. Majda, Eds. New York: Springer-Verlag, 1987.

[17] M. Gage and R. S. Hamilton, "The heat equation shrinking convex plane curves," J. Differential Geometry, vol. 23, pp. 69-96, 1986.

[18] I. M. Gelfand and S. V. Fomin, Calculus of Variations. Englewood Cliffs, NJ: Prentice-Hall, 1963.

[19] M. Grayson, "The heat equation shrinks embedded plane curves to round points," J. Differential Geometry, vol. 26, pp. 285-314, 1987.

[20] A. Gupta, L. von Kurowski, A. Singh, D. Geiger, C. Liang, M. Chiu, P. Adler, M. Haacke, and D. Wilson, "Cardiac MRI analysis: Segmentation of myocardial boundaries using deformable models," Siemens Corp. Res., Princeton, NJ, Tech. Rep., 1995.

[21] S. R. Gunn and M. S. Nixon, "A dual active contour model," BMVC'94, Sept. 1994, pp. 305-314.

[22] G. Huisken, "Flow by mean curvature of convex surfaces into spheres," J. Differential Geometry, vol. 20, pp. 237-266, 1984.

[23] M. Crandall, H. Ishii, and P. L. Lions, "User's guide to viscosity solutions of second-order partial differential equations," Bull. Amer. Math. Soc., vol. 27, pp. 1-67, 1992.

[24] M. Kass, A. Witkin, and D. Terzopoulos, "Snakes: Active contour models," Int. J. Comput. Vision, vol. 1, pp. 321-331. 1987.

[25] S. Kichenassamy, A. Kumar, P. Olver, A. Tannenbaum, and A. Yezzi, "Gradient flows and geometric active contours," in Proc. ICCV, June 1995, Sept. 1994.

[26] , "Conformal curvature flows: From phase transitions to active vision," Arch. Rational Mech. Anal., vol. 134, pp. 275-301, 1996.

[27] B. B. Kimia, A. Tannenbaum, and S. W. Zucker, "Toward a computational theory of shape: An overview," in Lecture Notes in Computer Science, vol. 427. New York: Springer-Verlag, 1990, pp. 402-407.

[28] B. B. Kimia, A. Tannenbaum, and S. W. Zucker, "Shapes, shocks, and deformations-I," Int. J. Comput. Vision, 1995.

[29] B. B. Kimia, A. Tannenbaum, and S. W. Zucker, "On the evolution of curves via a function of curvature-I: The classical case," J. Math. Anal., Applicat., vol. 163, pp. 438-458, 1992.

[30] R. J. LeVeque, Numerical Methods for Conservation Laws. Boston: Birkhäuser, 1992.

[31] P. L. Lions, Generalized Solutions of Hamilton-Jacobi Equations. Boston: Pitman, 1982.

[32] R. Malladi, J. Sethian, and B. Vemuri, "Shape modeling with front propagation: A level set approach," IEEE Trans. Pattern Anal. Machine Intell., vol. 17, pp. 158-175, 1995.

[33] F. Morgan, Riemannian Geometry. Boston: John and Bartlett, Boston, 1993.

[34] T. McInerney and D. Terzopoulos, "Topologically adaptable snakes," in Proc. ICCV'95, June 1995, pp. 840-845.

[35] P. Neskovic and B. Kimia, "Three-dimensional shape representation from curvature-dependent deformations," LEMS, Brown Univ, Tech. Rep. 128, 1994.

[36] P. Olver, G. Sapiro, and A. Tannenbaum, "Geometric invariant evolution of surfaces and volumetric smoothing," SIAM J. Math. Anal., submitted for publication.

[37] S. Osher, "Riemann solvers, the entropy condition, and difference approximations," SIAM J. Numer. Anal., vol. 21, pp. 217-235, 1984.

[38] S. J. Osher and J. A. Sethian, "Fronts propagation with curvature dependent speed: Algorithms based on Hamilton-Jacobi formulations," J. Computat. Phys., vol. 79, pp. 12-49, 1988.

[39] S. Osher and L. I. Rudin, "Feature-oriented image enhancement using shock filters," SIAM J. Numer. Anal., vol. 27, pp. 919-940, 1990.

[40] P. Perona and J. Malik, "Scale-space and edge detection using anisotropic diffusion," IEEE Trans. Pattern Anal. Machine Intell., vol. 12, pp. 629-639, 1990. 
[41] M. H. Protter and H. F. Weinberger, Maximum Principles in Differential Equations. New York: Springer-Verlag, 1984.

[42] S. Raganath, "Contour extraction from cardiac MRI studies using snakes," IEEE Trans. Med. Imag., vol. 14, pp. 328-338, 1995.

[43] G. Sapiro and A. Tannenbaum, "On invariant curve evolution and image analysis," Indiana Univ. J. Math., vol. 42, 1993.

[44] G. Sapiro, A. Tannenbaum, and Y. L. You, "Experiments geometric image enhancement," presented at Proc. 1st Int. Conf. Image Processing, Austin, Texas, 1994

[45] J. A. Sethian, "An analysis of flame propagation," Ph.D. Dissertation, Univ. California, 1982.

[46] , "Curvature and the evolution of fronts," Commun. Math. Phys., vol. 101, pp. 487-499, 1985.

[47] _ "A review of recent numerical algorithms for hypersurfaces moving with curvature dependent speed," J. Differential Geometry, vol 31, pp. 131-161, 1989.

[48] J. A. Sethian and J. Strain, "Crystal growth and dendritic solidification," J. Computat. Phys., vol. 98, 1992.

[49] J. Shah, "Recovery of shapes by evolution of zero-crossings," Dept. Mathematics, Northeastern Univ., Boston, MA, Tech. Rep., 1995.

[50] J. Smoller, Shock Waves and Reaction-Diffusion Equations. New York: Springer-Verlag, 1983.
[51] M. Spivak, A Comprehensive Introduction to Differential Geometry. Berkeley, CA: Publish or Perish, 1979.

[52] R. Szelski, D. Tonnesen, and D. Terzopoulos, "Modeling surfaces of arbitrary topology with dynamic particles," in Proc. CVPR, 1993, pp. $82-87$.

[53] H. Tek and B. Kimia, "Deformable bubbles in the reaction-diffusion space,” LEMS, Brown Univ., Tech. Rep. 138, 1994.

[54] D. Terzopoulos and R. Szelski, "Tracking with Kalman snakes," in Active Vision, A. Blake and A. Zisserman, Eds. Cambridge, MA: MIT Press, 1992.

[55] D. Terzopoulos and A. Witkin, "Constraints on deformable models: Recovering shape and nonrigid motion," Artificial Intell., vol. 36, pp. 91-123, 1988.

[56] R. Whitaker, "Algorithms for implicit deformable models," in Proc. ICCV'95, June 1995, pp. 822-827.

[57] B. White, "Some recent developments in differential geometry," Mathematical Intelligencer, vol. 11, pp. 41-47, 1989.

[58] G. Xu, E. Segawa, and S. Tsuji, "Robust active contours with insensitive parameters," Pattern Recog., vol. 27, no. 7, pp. 879-884, 1994

[59] A. Yezzi, A. Tannenbaum, S. Kichenasamy, and P. Olver, "A gradient surface approach to 3-D segmentation," in Proc. IS\&T 49th Annu. Conf., Minneapolis, MN, May 1996. 